\title{
'T'II E
}

\section{CAMBRIDGE LAW JOURNAL}

EDITED BY

H. A. HOLLOND, M.A., LL.M.

AND

P. H. WINFIELD, LL.D.

FOR

THE CAMBRIDGE UNIVERSITY LAW SOCIETY.

VOL. III.

LONDON

STEVENS \& SONS LIMITED

1929 
Published by Stevens \& Sons

Limited of 11 New Fetter Lane

London, Great Britain - Law

Publishers - and reprinted in

1962 by photolithography in The

Netherlands by Krips/Oosthoek

of Rijswijk, Utrecht and London

Printed in The Netherlands 


\section{TABLE OF CONTENTS.}

\section{INDEX OF SUBJECTS.}

PAOS

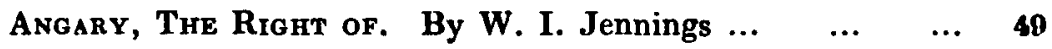

Appeal in English Law. By the Right Hon. Lord Justice

$\begin{array}{llllllllll}\text { Atkin } & \ldots & \ldots & \ldots & \ldots & \ldots & \ldots & \ldots & \ldots & 1\end{array}$

Bacon. By Prof. W. S. Holdsworth $\ldots$.

Book Reviews $\quad \ldots \quad \ldots \quad \ldots \quad 118-147,303-354,466-498$

Cases, Notes on Recent ... $\quad \ldots \quad 75-112,240-303,418-466$

Common Law, Thoughts About the. By the Right Hon.

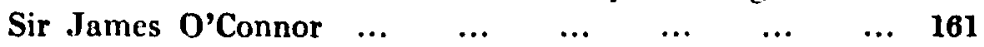

Corporations, Criminal Regponsibility of. By C. R. N.

$\begin{array}{lllllllll}\text { Winn, LI.M. } & \ldots & \ldots & \ldots & \ldots & \ldots & \ldots & \ldots & 898\end{array}$

Dangerous Tuxges and the Non-Natural User of Land.

By W. T. S. Stallybrass, Fellow of Brasenose College,

$\begin{array}{llllllllll}\text { Oxford } & \ldots & \ldots & \ldots & \ldots & \ldots & \ldots & \ldots & \ldots & \mathbf{8 7 6}\end{array}$

Droit Administratif. By Prof. Achille Mestre $\ldots \quad \ldots \quad 855$

Equitable Liens Under Hire-Purchage Agreements. By

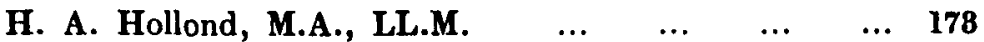

Imperial Conference and the Constitution. By Prof.

$\begin{array}{lllllllll}\text { Edward Jenks } & \ldots & \ldots & \ldots & \ldots & \ldots & \ldots & \ldots & 18\end{array}$

Irish Bar, The Last Forty Years of the. By Serjeant

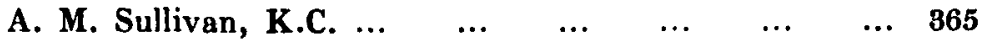

Johnson, Dr., and the Old Bailey. By Theobald Mathew 182

Law Student, The Happy State of the Modern. By the

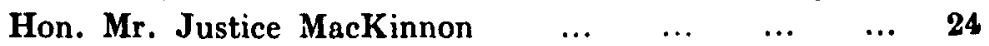

Mandates. By Arnold D. McNair, C.B.E., LL.D. ... $\quad \ldots \quad 149$

Parliament and the Dominions: A Retrospect. By Prof.

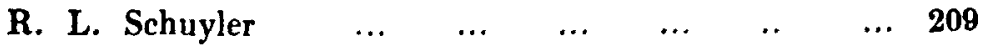


Personality of an Idol, The. By P. W. Duff, Fellow of $\begin{array}{lllllll}\text { Trinity College, Cambridge } & \ldots & \ldots & \ldots & \ldots & \ldots & 42\end{array}$

Philosophy of Law, Some Reflections on the. By Sir

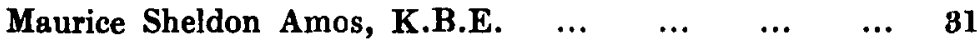

Possegsion, Three Cases on. By A. L. Goodhart, M.A.,

$\begin{array}{llllllllll}\text { LL.M. } & \ldots & \ldots & \ldots & \ldots & \ldots & \ldots & \ldots & \ldots & 195\end{array}$

Trusts for Sale. By J. M. Lightwood, sometime Fellow of $\begin{array}{lllllllll}\text { Trinity Hall } & \ldots & \ldots & \ldots & \ldots & \ldots & \ldots & \ldots & 59\end{array}$ 


\section{$(v)$}

\section{INDEX OF CONTRIBUTORS.}

Amos, Sir M. S.-Some Reflections on the Philosophy of Law $\ldots 31$

ATKIN, The Rt. Hon. Lord Justice._Appeal in English Law $\ldots 1$

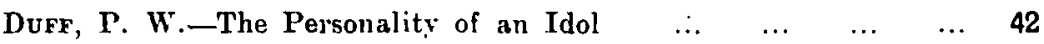

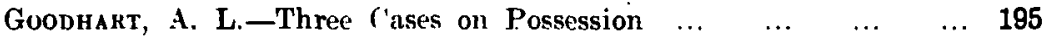

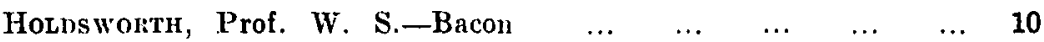

Hollond, H. A.-Equitable Liuns Under Hire-Purchase Agreements 173

Jenks, Prof. Edward.-CThe Imperial Conference and the Constitution 13

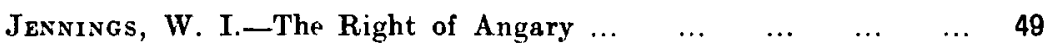

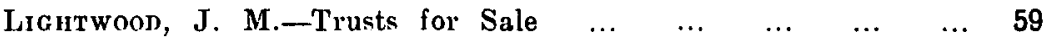

MacKinnos, The Hon. Mr. Justice.-The Happy State of the

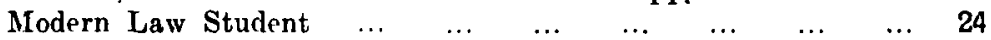

$\begin{array}{llllllllll}\text { McNaIr, A. D. Mandates } & \ldots & \ldots & \ldots & \ldots & \ldots & \ldots & \ldots & 149\end{array}$

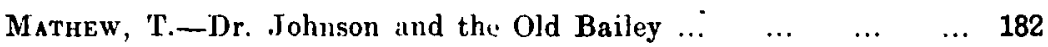

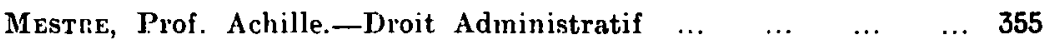

O'Coxwon, The Rt. Hon. Sir .James.-Thoughts About the Common

$\begin{array}{lllllllllllll}\text { Law } & \ldots & \ldots & \ldots & \ldots & \ldots & \ldots & \ldots & \ldots & \ldots & \ldots & 161\end{array}$

Schuylek, Prof. R. L.-Parliament and the Dominions: A

$\begin{array}{lllllllllll}\text { Retrospect } & \ldots & \ldots & \ldots & \ldots & \ldots & \ldots & \ldots & \ldots & \ldots & 209\end{array}$

Stallybrass, W. T. S.-Dangerous Things and the Non-Natural

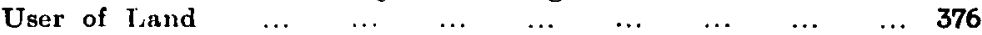

Suldrvas, Serjeant A. M., K.C.-The Last Forty Years of the Irish

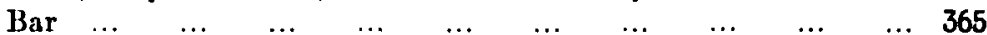

Wins, C.R.N.—Criminal Responsibility of Corporations $\ldots{ }^{2} \quad \ldots 398$ 


\section{TABLE OF CASES.}

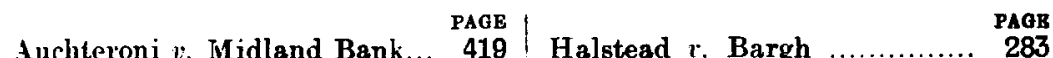

Auchleroni

Hardie \& Lane $v$. Chilton ... 436

Behnke v. Bede Shipping Co. 264

Hilton v. Westminster Bank... 87

Blackwell, Re 302, 459

Board of Trade $v$. Cayzer, etc. 268

Bohemian Union Bank v. Administrator of Austrian Property

Hodson v. Barnes

103

Hong Kong, etc. Corporation $v$.

Lo Lee Shi

242

Horton's Estate $v$. James Beattie .................... 90

Britt v. Galmoye

249

Brooke v. Bool

455

Broome v. Agar

456

Buckle v. Holmes

451

Bull, A. H. v. W. African Shipping, etc. Co.

Burrel $v$. Levin 288

79 Importers' Co. $v$. Westminster

$$
\text { Bank ........................ } 240
$$

Ganadian Pacific Ry. Co. $v$.

Kelvin Shipping (o. ......

Chardon, lie ......................

(Yeghorn $x$. Oldham .............

Cohen $v$. Roche .................

('oleshill v. Manchester ('orporation

Crown Milling Co. $v$. R.

458

463

294

267

Inche Noriah $v$. Shaik, etc. ... 440

Ironmonger $v$. Dyne .......... 260

Johnston $v$. Davies ............. 463

Jones, R. E., Ltd. $v$. Waring

\& Gillow 83,84

290

275

Collins v. Henry Whiteway ... 292

Jupiter. The

80

Kasler $r$. Slavouski ........... 270

Keppel $v$. Wheeler ............ 257

Davis $v$. Mayhe $:$............ 297

Kettle $v$. Dunster ............... 243

Koechlin $v$. Kestenbaum ..... 241

Ellesmert r. Wallace .......... 429

Konskier v. Goodman .......... 295

Engelke r. Musmann .......... 441

English Hop Growers $v$. Dering 433

Eshughayi Eleko $v$. Officer, etc.

Fagernes, The ............... 277

426

Farr \& Co. $r$. Messers. ........ 266

Ford, $R, \ldots \ldots \ldots \ldots \ldots \ldots, 478$

G. W. K. v. DunloJ' Rubber Co.

Glanville $r$ Sutton ............ 287

Gray v. Perpetual Trustee Co. 461

Greel r. Downs Supply Co. ... 259

Lanyon, The ................... 299

Laubach v. Co-optimists' Syndicate .................. 93

Leigh's Settled Estates, Re ... 110

Letang $v$. Ottawa Electric Ry. Co. $\ldots \ldots \ldots \ldots \ldots \ldots \ldots \ldots . . . \ldots 8$

Lloyds Bank v. Chartered Bank, etc. ................ 423

Lockhart $\imath$. Harrison ......... 452

Looker $v$. Law Union, etc., Co. 430

Lowther $v$. Harris ............. 101 


\begin{tabular}{|c|c|c|c|}
\hline \multirow{2}{*}{$\begin{array}{l}\text { Mackenzie-Kennedy } \quad v . \quad A \mathrm{ir} \\
\quad \text { Council }\end{array}$} & & 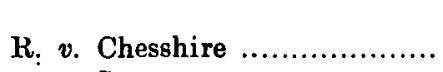 & \multirow{2}{*}{$\begin{array}{r}\text { PAGR } \\
272 \\
274\end{array}$} \\
\hline & 252 & - v. Cory & \\
\hline Marbe $v$. George Edwardes ... & 253 & -v. Whitehead .. & 38 \\
\hline 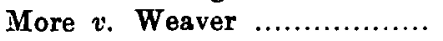 & 450 & Reckitt $v$. Barnett .............. & 445 \\
\hline lli $v$. Fitch .................. & 447 & Rely-a-Bell, etc., Co. $v$. Eisler & 103 \\
\hline In tho Fototo & 297 & $\begin{array}{l}\text { Richardson v. Moncrie } \\
\text { Rowland, etc. } v \text {. Air Cou }\end{array}$ & $\begin{array}{r}78 \\
252\end{array}$ \\
\hline $\begin{aligned} \text { ational } & \text { Sailors' Union } r \\
\text { Reed } & \ldots \ldots \ldots \ldots \ldots \ldots \ldots \ldots \ldots\end{aligned}$ & 99 & $\begin{array}{c}\text { Royal London Insurance } \\
\text { Society } v \text {. Barrett ............. }\end{array}$ & 431 \\
\hline oble $v$. Harrison $\ldots \ldots \ldots \ldots$ & & $\begin{array}{l}\text { St. Anne's Well Brewery Co. } \\
\text { v. Roberts } \ldots \ldots \ldots \ldots \ldots \ldots\end{array}$ & \\
\hline gle's Settled Estates, $R e \ldots$ & 280 & $\begin{array}{l}\text { therley } \ldots \ldots \ldots \ldots \\
e \ldots \ldots \ldots \ldots \ldots \ldots\end{array}$ & \\
\hline $\begin{array}{l}\text { almolive Co. } v . \text { Freedman ... } \\
\text { arker } v \text {. Miller ................. } \\
\text { arker's Settled Estates, } \text { Re ... }\end{array}$ & $\begin{array}{r}434 \\
95 \\
281\end{array}$ & $\begin{array}{l}\text { Terry } v . \text { Variety Theatres ....... } \\
\text { Tomkins } v \text {. Coates .................. }\end{array}$ & $\begin{array}{r}427 \\
88\end{array}$ \\
\hline 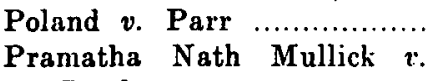 & 91 & $\begin{array}{l}\text { Villar, Re } \\
\text { Visser, } R e .\end{array}$ & $\begin{array}{l}465 \\
443\end{array}$ \\
\hline ablic Trustee $v$ Ville & 46 & $\cdots \cdots$ & \\
\hline
\end{tabular}

